\title{
ON THE INTUITIVE GEOMETRY
}

Osvaldo Rojas ${ }^{1}$, Javier González ${ }^{2}$, Mauro García ${ }^{3}$

${ }^{1}$ Professor and researcher of the PhD and Master programs of Mathematics Education.

University Antonio Nariño (UAN), Bogotá, Colombia, Nicolás de Federman, Bogotá, Colombia.

$$
\text { orojasv2301@gmail.com }
$$

${ }^{2}$ Professor of the Department of Mathematics at the University Autónoma de Guerrero, Guerrero, Mexico.

jg_mendieta@hotmail.com

${ }^{3}$ Director of the $\mathrm{PhD}$ and Master program of Mathematics Education. University Antonio Nariño (UAN), Bogotá, Colombia, Nicolás de Federman, Bogotá, Colombia.

mauro@uan.edu.co

\section{ABSTRACT}

In this paper the origin of the concepts of geometry and the way and the reason for systematization is analyzed. A first approach to the analysis of the processes involved in the development of geometry determines its target and leads us to clarify what is meant by a mathematical object and, further, what is meant by geometric object, and leads into the structure essential geometry.

\section{Keywords}

Geometry, Intuitive Geometry, Object, Math

\section{Academic Discipline And Sub-Disciplines}

Education, Teaching of Mathematics Education

\section{SUBJECT CLASSIFICATION}

Innovation in education

\section{Council for Innovative Research}

Peer Review Research Publishing System

\section{Vol. 7, No.3}

Journal: International Journal of Research in Education methodology

iiremeditor@gmail.com

www.ijrem.com 


\section{INTRODUCTION}

We ask Each generation adds knowledge to that already accumulated, if not so we would always have to start over, this creates the opportunity to conduct studies that otherwise would too long for a human to carry out, so the work is taken up, again and again, by new generations, who are better off culturally, scientificly and technologicaly to do it. Hence, knowledge is the result of the work of many generations, consciously or unconsciously, with the use of writing or not, accumulating a huge amount of information related to the forms and structures of objects and is now called Geometry .

More than a million and a half years ago, a time period now called prehistory ranging from the appearance of man until the invention of writing, was the forging scene of countless intuitive ideas of geometry, that is to say Paleogeometry. The writing determines a total and advanced intellectual level, we began, as a species, to give a purely ideographic sense to the drawings to become a first writing system, but the drawings came to represent ideas, which are now not the drawings that essentially characterize them; an intellectual level in the culture is thereby reached; whenever a group of humans accepts that the same drawing represents an object or idea it will be able to accept not just one but a set of images that are ordered, thus constituting a pictographic script and then unequivocally to the laws of the written language.

The search for the origins of geometry is uncertain, is deeply tied to the origin of humanity, the analysis of prehistory and comparison of knowledge of "geometry" of the previous socio-cultural groupings which are long gone remains to be accomplished. In any case, to study the origin of geometry is equivalent to studying the origin of culture, civilization, studying the evolution and development of geometry is closely linked to the study of the history of scientific and technological development of mankind; but studying the history of geometry is not the same as studying the causes that gave rise to it; these are two well defined problems and require a different analysis. The origin of geometry leads us toward an epistemological view, associated with the theory of knowledge, which for completeness in the study goes along with historical and logical analysis of it.

The original intent of the geometry was to study the forms, spatial configurations and the relationships between natural bodies; This leads us to consider that, they began by determining the relationships between their different spatial properties, such as volume, surface, number of faces and physical characteristics, such as weight and density, among others, since it was not distinguished between physical and geometric properties. It did not begin by studying the characteristics of the bodies as isolated properties, rather, everyday problems led to the study of these properties.

Thus, geometry grew out of the observation of the natural world, interaction that humans have always had with it, and because of this, geometry has become more extensive and practical. Where else, but it is the observation of the natural world that we reached our first ideas? Where else, if the world recharge all our senses here and now? which, as a result, we can abstract, particularize, invest, compare, make metaphors and thought experiments that lead us, in a Word, to reflect, to seemingly unimaginable concepts that we can not easily recognize in nature, abstractions that do not appear to have a connection with the natural world and, however, they are logical; then, and from these first observations, emerged the first applications of geometry, measurement of land, the construction of temples, tombs and pyramids, roads and tools that made life easier, the existence of tools that made the work easier and that increased supplies and wealth.

Geometric elements have been present in the development of human civilization, they are not the result of a single thought, but a collective consciousness, entrenched in the past 300,000 years. It is clear that it will always be a process of approximation trying to know the year in which the cavemen learned how to count, the same is true with the discovery of the use of fire and the wheel. They were discoveries that made many prehistoric groups and which they must have used when their needs grew; Thus, we perhaps have a clue, the need creates the basis of the invention. We can imagine a "caveman genius", who realized, for the first time that on soaking logs with tar fire lasted much longer and that it was not easily extinguished by the rain, we can imagine him trying to carry a trunk to his cave and discovering in doing so that rolling it is easier; but in imagining this we must not forget that its invention was due to a need, it was not isolated but collective, there were probably many who labored to bring logs to the cave or who needed the fire to last longer, ultimately their minds evolved, intuitively understanding the geometric essence of the problem, in short, it improved their reasoning and they understood the world better.

Why can we analyze the world that surrounds us in geometric form? Our mind has evolved according to the geometry of the natural world, there is an interaction between the brains of living beings that modifies and is modified by the object in question. If our everyday world had a hyperbolic geometry or the immediate natural physical laws were quantum perhaps then our brains would have become accustomed to these laws, we would have evolved according to them, they would not seem strange to anyone and our classical geometry would be the non-Euclidean. We construct our geometry according to the natural world and the natural world to our geometry, our "immediate reality" is that of the "classical physics", for this reason we evolved that way, our brain has adapted to nature and not it to us. Moreover, we don't have reason to think that the natural world is one and we are independent of it, we are part of it and with it we evolved at the same time; that is why our geometric mind evolved together with the geometry of nature; We do not adapt the geometry of the natural world or modify it, our mind did not modify nature, we are a whole, a unique multivariate world and all interacting in all.

A study of the beginning of the culture of the primitive man, determined to find the origins of geometry, and the same happens with the study of the behavior of animals with regard to mathematics, and particularly geometry. Under these aspects, you must delimit the development of primitive geometry. Neanderthals were nomadic, did not leave many traces in the geometric aspect, so we have to guess about their notions of geometry based only on cave paintings and the remains of its buildings and first-time shelters. 
To the prehistoric men, learning to count must not have been easy, neither were they aware of this, the new ideas concerning number were only empirical. Indeed, the first truly serious attempts to respond to questions about the concept of number, sum and product were very much later, and the formal definition of integer, sum of integers and others, apparently elementary, were given up to the nineteenth century (1889) by Giuseppe Peano (1858-1932) and F. L. Gottlob Frege (1848-1925), and then taken up once again by Bertrand Russell (1872-1970) among others who systematized this aspect of mathematics.

\section{The development of geometry}

To understand the development of science and in particular geometry, three aspects involving the so-called levels of conceptualization of mathematical must differ. First, we must consider the rise or origin of the concepts of geometry; Second, the development of concepts or Ideas involved and, third, the formalization of these concepts; three aspects of importance that clarify the study of concepts of geometry, its origin, its development and its formalization. Simultaneously with the process of counting, the measurement process was developed, probably prehistoric men made their first measurements in an improvised way, comparing certain parts already known to them, at the beginning were parts of their own bodies, the foot, the length of the arm and perhaps the extent of the steps. To measure distances we always compare two lengths, one of which is designated as unit of measure; Likewise, when measuring speeds compared the speed of the runners, the fastest can serve as a unit of measure, and also compares the size of animals with regard to one of them or in relation to our stature, may be the unit of measurement. We can also measure other things, intelligence, visual acuity, skill and even more complex things, but the unit of measurement is always arbitrary and eventually becomes conventional.

When we measure a distance $d$ between two objects, what we do is compare how many times is the unit of measure $u$ on the length $\mathrm{d}$, distance meaning the degree of rapprochement between two objects; in other words, how close is one to the other. Really, to measure is to compare; If $d$ represents a length and or the unit of measure then we say that $u$ measured at $d$ if there is an integer $n$ such that: $d=n u$, with $n \in \mathbb{N}$, if the number $n$ does not exist then we can make subdivisions of $u$; that is to say $u / m, m=2,3,4, \ldots$ and again find $n$, and therefore $d$ can be represented in the form, if we can do this again we say that $u$ measured at $d$, otherwise we can repeat the process again, i.e. dividing one of the $m$ parts $u$ in subparts, regularly spaced and this, approach the measuring as much as you want again writing to $d$ in the form; However, eventually it could happen that there is no subdivision of $u$ to determine $d$, in which case we can consider that there is a remainder of $c$, deficiency or excess, to be determined, with which we can represent $d$ in the form, $n, m \in \mathbb{N}$ and $c \in \Re$, which leads to the need to clarify the nature of $c$, and the set of numbers $\Re$.

The magical and mystical experiences led to the recognition of geometric figures, such as circles, straight lines, planes and spheres; geometric shapes that can be found in the anatomy of animals, the phases of the Moon, the Sun, the waves of water, in the rays of light filtering through the trees, in the Rainbow and even in the eyes of humans. Contemplation of the harmonious aspects of the natural world must awaken the curiosity of old men and the approach to the reflection of that beauty like no other. The symmetry, the multiplicity and the regularity of the processes and the colors made them feel and appreciate the beauty of the natural world. And, of course, the initial curiosity led them to try to make ever better sense of the variety of geometric forms in nature.

The sensations, emotion and joy produced by the beauty of the objects, are directly related to the conscious state of the subject and are represented, hence the geometric transformation that man reflected in his paintings, geometric alterations that do not seem to exist in nature must have arisen from there. This aesthetic pattern was repeated for thousands of years and, without their being aware of it, primitive men developed the idea of the straight line, circle and other shapes, which were represented in numerous paintings. Perhaps at this point, it is necessary to clarify the idea of imagination in relation to primitive man, in that we need to explain the development of geometry in our minds.

Some anthropologists have expressed that primitive man, such as the man of Toumai, from a time of 6 or 7 million years ago, and the Australopithecines, lacked imagination. Today, in no way, can we imagine how and where we will be tomorrow, and not only that, we can [only] imagine what instruments we will need to be much better off. If we want to go for a ride, we can imagine how it would be, what we should bring, what we should buy and we can even imagine driving a car to get to a Lake to fish at, and even more, we can visualize ourselves, at the gas station, filling the fuel tank, something that apparently has nothing to do with fishing; that is, we can imagine much more of what is today's world; and let alone how it is that we imagine what we need to solve a complex problem of geometry, the way, in our minds images that represent the possible solution come about.

But primitive man could not imagine all that, there are anthropomorphic arguments to this effect! If one of the members of the tribe dyed they simply threw him out of the cave or, even worse, they ate him. How did they imagine where he went? The imagination, as explained by anthropologists and paleontologists, emerged much later, perhaps with Neanderthal or Cro-Magnon man. Is this what characterizes the human species, the ability to imagine geometric shapes, among many other things? The oldest paintings are from the Paleolithic period, 35,000 to 30,000 years BC (Aurignac, France). Not less than 2,000 paintings have been studied in Spain, France and in general in Europe, confirming the variety of styles, materials used, and figures in paintings; among the most sophisticated are: Teyat, Lascaux, between 13,000 and 14,000 years b.c. Les Trios Frères, Les Convarelles in France, the Castillo and Altamira in Spain. We also found them in America; on the peninsula of Baja California, Mexico, not less than 200 sites have been found with cave paintings dating between 12,000 to 8,000 years old.

Against this background, it is necessary to reflect on the fact that, since the beginning of the history of artists, from the time of first cavemen until that of more sophisticated painters, their drawings have always depicted three dimensional figures on a two dimensional surface. And that has ultimately led to the development of a study of the perspective that 
originated in the early cultures, then the great ancient civilizations, Mesopotamian, Egyptian, Greek and Hindu set the foundation for its development and, thereby, to lead, in the 15th century, to the study of perspective and projective geometry which had a boom with the artists of the Renaissance period and hence to the representation of threedimensional images on screens or monitors of electronic computers.

Architecture, seen here as the construction of huts and new homes, has also contributed to the origin of geometry; the first huts that were built by the primitive men show the use of countless geometric elements, for example, in North Africa, the construction of the first huts was developed based on circles, boxes, and essentially symmetrical arrangements. Likewise, remains have been found, in the North of Canada, of circular constructions that bear witness to the fact the ease, strength and versatility of a refuge with this shape, built even with arched ceilings, arches that are nothing more than mammoth tusks, walrus or Whale bones. Observation of convenient geometric forms and some intuition about the resistance of the materials motivated primitive men to build with more selective geometric shapes. Thus arose geometry, i.e. subconsciously, but effective in the fight for survival. Then huge megalithic constructions appeared made of huge blocks of stone that led to very sophisticated geometric shapes; and religion and superstition was also a great incentive for the collective construction of countless temples and monuments and, therefore, astronomy was one ingredient in the development and consolidation of geometry. To what degree did astronomy influence the origins of geometry?

After the first buildings, primitive men didn't take long in extending its interest to the sky, trying to find harmony and regularity in the sky they became engaged in the study of the cosmos. At the beginning the study was not systematic and took a lot of time in organizing Recognizing shapes and patterns of regularity in it, they obtained data that, although having no real meaning, helped to create the necessary basis for future interpretations. With imagination, men of old, put figures and magical beings in the sky, created legends about them, assigning a mystical and religious significance to the study of the sky. Figures or constellations, which we recognize in the sky are not really there, they are imposed by the mind that "sees" the intrinsic geometry; We can not manipulate stars, but we can do it with imagination, that is the true geometric power of the human mind.

From ancient times witches, sorcerers and astrologers of the first tribes sought to understand the sky in order to learn about their "divine laws", thus obtaining power over the common man who did not understand anything related to the sky, accept its strange beauty. To understand this let us imagine, just for a moment, the power of those who could predict the date and perhaps the time of a solar eclipse. What a feeling of horror for the villagers to see that day becomes night. What idea would they have of those, the prophetic witches, who predicted it, the "Prophecy" after the event? They became representatives on Earth of the gods! Those who predict spectacular astronomical events had the power. But, how could they predict solar eclipses? How could they develop a proper calendar? Was something so complicated to be predicted in advance? Many times the ancients were not even able to explain what was happening and their methods were the result of many traditions, but they worked! and that was important to be able to continue in the right direction. Thus was born astrology, that gave birth to astronomy, and there can be no astronomy without geometry, since trying to do a study of the sky without geometry leads to a total disorder in the understanding of the heaven and the laws of cosmic nature.

Impressive also, is the way that Stonehenge was built, as some rocks which form it weigh 200 tons, it must not have been easy, but required geometry, a lot of geometric observation at least, as the stone age men must have at least traced out the path to where these rocks (dolmens and menhirs) were moved. Apparently those who built Stonehenge knew more geometry than what we assume they knew and this is almost always the case. This was perhaps the beginning of a new race, set on perfection, which gave rise to the empirical geometry. So far, everything points in the direction that geometry arose in its relationship with nature; However, tsaying it that way it seems as if it we were saying that geometry was in nature. The geometry is not immersed in nature; of course, although, arguably, $\pi$ is in the trunk of a tree and it is also in the circle of the Sun or the full moon and, looking closer, it is in many places, but really $\pi$ is nature?... In the beginning $\pi$ was understood as the ratio of the length of the circumference and its diameter, but we make the comparison or measurement between the length of the circumference and the diameter not nature!

But of course, the higher you want to go, the greater base should be and there is where the platforms and the Structural geometry of the pyramid are required which leads to a geometry problem; that same method used with the first pyramids of Egypt; at the beginning were little elegant platforms on platforms such as the Sakara in the Sahara, and then they put more and more platforms, and unintentionally, the sides became increasingly more continuous, until refined pyramids of Gizeh, were obtained and motivated by a religious and mystical longing, constructions became pyramidal; in the end a structurally necessary geometry, and at the same time a primitive geometry, the pyramids, unfolded.

A deeper analysis of the emergence of the Geometry makes us reflect on the evidence we have of its origin and its authenticity and, moreover, the interpretation we make of it; modern techniques of Archeology and Paleontology offer little data regarding this and all these are interpreted from our current geometric perspective. We can only interpret paintings and drawings that prehistoric man left under our present historical and cultural context and modern scientific perspective. And with all this, it may be that we do not understand the real reasons why civilizations made such and such thing, we can only, to a certain extent, assume and venture into the interpretation of the origin of their geometric ideas, in any case, it is an interpretation; however, the analysis and extensive works made by epistemologists, psychologists and sociologists establish a frame of reference from which we conduct our analysis.

We are not the first to try to study primitive geometry, the ancient Greeks believed that it had its origin in ancient Egypt, more or less in the year $3000 \mathrm{bc}$, and that originated due to the need to re-measure the limits of the plots that were lost during the overflowing of the Nile River; and that later it was used in the construction of temples and pyramids. The construction of the pyramids of Gizeh shows the validity it of this assertion, since it was necessary for the construction of such impressive monuments. All this is fine if we consider that the great pyramids of Egypt were built 2600 years bc, but 
there are those who call into question validity of this assertion; Dr. Robert Schoch, of Boston University, the Egyptologist John West and engineer Robert Bauval claimed, in 1994 that the construction of the pyramids of Gizeh dates back to the year 12,500 b.c. which leads us to rethink the situation, and now we must consider that the geometry required for the construction of the great pyramids the Egyptian had to have developed much earlier, perhaps several thousand years earlier.

Geometry not only began because certain similarities between the different parts of the geometric objects were observed but also when humans were capable of structuring these similarities in a rational manner. And of course this doesn't mean that it is the only way of understanding the world. Geometry was later systematized and developed under this perspective.

At what moment did we come to distinguish between circle, sphere, cylinder and cone? At what point did we begin to distinguish objects of geometry? In the context of the construction of geometry. We can very well distinguish the historical event, perhaps the particular time, that ancient men began to use geometry, but it is not very clear when they realized that were developing, structuring, and systematizing geometry. All this leads us to think that, as a first approximation of what is meant by geometrical object and its relationship to abstraction, i.e. the recognition of the essential properties that designate a collection of objects of the world objective, but not only that, because if it were we would also have to include many other concepts such as the conscience, morality and much more. It must, then, be something more determined when an abstraction is mathematic and, more so when it is geometric.

The original objective of geometry was the study and systematization of knowledge related to the spatial forms of the objective world; however, if our reflection leads us to enter into the domain of geometry then, in what does study the geometry consist?... well, in part, no doubt, geometric objects; so then, how should we understand a geometrical object? and what is the objective of study of geometry? One must distinguish between a fundamental mathematical object and mathematical object. Fundamental mathematical object refers to an abstraction associated with quantitative or spatial relations of the objective world; and, therefore, fundamental geometrical object refers to an abstraction associated with the spatial relationships of the objective world. However, it should be made clear that this idea of fundamental mathematical object is fundamental, Since from it come generalizations, and more importantly, new abstractions, so, $\Re^{3}$ space is nothing more than a generalization about the fundamental geometrical object from Euclidean space which, in turn, is an abstraction of natural or objective space. Thus, the geometric objects of geometry in $\mathfrak{R}^{n}$ are subsets of $\mathfrak{R}^{\mathrm{n}}$ which are, again, a generalization or abstraction of abstract objects such as point, distance, line, surface and volume and, in general, of the geometric objects in Euclidean geometry that, in turn, are an abstraction of natural-space objects, with this we again fall into the generalization and abstraction made on abstractions which, in turn, are made on quantitative and spatial relations.

Once you recognize the fundamental mathematical abstractions generalizations or abstractions can be on them; Hence, mathematical object refers to a generalization on one or more abstractions which, in turn, must be connected with the abstractions of fundamental mathematic objects and, therefore, refers to a geometric object a generalization or abstraction of one or more abstractions which, in turn, must be connected to the abstractions of the fundamental geometric objects.

\section{CONCLUSIONS}

From all this it can be inferred that abstraction process is first the generalization, i.e., first determine the essential characteristics of an object which are then generalized; or in other words, first is the concept and then the definition, but from the concept you can determine a new abstraction and take it to a generalization, which determines a new concept. So, we cannot expect that the first human groups were able to recognize the existence of geometric objects, that on the other hand had lacked proof of existence, and even more, that were aware of the incipient systematization of them. All progress of the construction of geometry theory has been gradual, a few abstractions have given rise to others and so on, in a sequence that clearly has no end.

\section{ACKNOWLEDGMENTS}

Universidad Antonio Nariño

\section{REFERENCES}

[1] Morales, A., Nolasco, H., Henández, J. C. and Sigarreta, J. M. 2014. Methodology based on problem solving in the treatment of the concept of limit to infinity. International Journal of Research in Education Methodology 5(1) (2014). 542-550.

[2] Nolasco, H., Henández, J. C. and Sigarreta, J. M. 2014. Methodology for study of epistemological obstacules: similarity concept. International Journal of Man \& Information Tech 8 (2) (2014). 1323-1333.

[3] Cruz, M., Rojas, O. and Sigarreta, J. M. 2014. Ciencias Some historical on nombres pi. Básicas en Ingeniería 11 (6) (2014). 51-62.

[4] Sigarreta, J. M. 2013. Hyperbolicity in median graphs. Proceedings Mathematical Sciences 123 (4) (2013), $455-467$.

[5] Sigarreta, J. M. 2011. Upper alliances in graphs. Int. J. Cont. Math. Sci. (6) (43) (2011). 2121- 2128.

[6] Behnke, H. 1988. Fundamentals of Mathematics. Volume II. MIT.

[7] Pedoe, D. 1976. Geometry and the Visual Arts. New York, Dover. 